\title{
The Abscisic Acid Pathway Has Multifaceted Effects on the Accumulation of Bamboo mosaic virus
}

\author{
Mazen Alazem, ${ }^{1,2,3}$ Kuan-Yu Lin, ${ }^{1}$ and Na-Sheng Lin ${ }^{1,2,4}$ \\ ${ }^{1}$ Institute of Plant and Microbial Biology, Academia Sinica, Taipei 115, Taiwan, ROC; ${ }^{2}$ Molecular and Biological Agricultural \\ Sciences Program, Taiwan International Graduate Program, National Chung-Hsing University and Academia Sinica; \\ ${ }^{3}$ Graduate Institute of Biotechnology, National Chung-Hsing University, Taichung, 402, Taiwan, ROC; ${ }^{4}$ Biotechnology Center, \\ National Chung-Hsing University
}

Submitted 8 August 2013. Accepted 29 October 2013.

\begin{abstract}
Abscisic acid (ABA) plays a key role in modulating plant responses to different biotic and abiotic stresses. However, the effect of $\mathrm{ABA}$ on virus infection is not fully understood. Here, we describe the effects of the $A B A$ pathway on the accumulation of Bamboo mosaic virus (BaMV) and Cucumber mosaic virus (CMV) in two different hosts: Arabidopsis thaliana and Nicotiana benthamiana. We report that $A B A 2$ plays a critical role in the accumulation of BaMV and CMV. Mutants downstream of $A B A 2$ (aao3, abil-1, abi3-1, and $a b i 4-1)$ were susceptible to BaMV, indicating that the ABA pathway downstream of $A B A 2$ is essential for BaMV resistance. The $a b a 2-1$ mutant decreased the accumulation of BaMV (+)RNA, (-)RNA, and coat protein, with the most dramatic effect being observed for (-)RNA. These findings were further validated by the use of virus-induced gene silencing and enzyme-linked immunosorbent assay in $N$. benthamiana. In addition, infecting $N$. benthamiana with BaMV or CMV increased ABA contents and activated the SA and ABA pathways, thereby disrupting the antagonism between these two cascades. Our findings uncover a novel role for $A B A 2$ in supporting $B a M V$ and $C M V$ accumulation, distinct from the opposing role of its downstream genes.
\end{abstract}

Plant hormones are small signaling molecules that regulate growth and development. Although they exist in small amounts, changes in their concentrations affect adaptive plant responses to biotic or abiotic stresses. Plant responses to biotic stress are controlled by the following hormone-regulated defense pathways: salicylic acid (SA), jasmonic acid (JA), and ethylene (Et) (Pieterse et al. 2009; Santner et al. 2009). These pathways antagonize one another and, thus, plants can finetune the levels of cross-talk to maintain effective defense while minimizing the metabolic cost. Plant resistance to viruses is usually mediated by the SA pathway. Upon activation, this pathway induces several defense responses, including reactive oxygen species (ROS) production, the hypersensitive response, and pathogenesis-related (PR) protein synthesis (Jovel et al. 2011; Lewsey et al. 2009; Soosaar et al. 2005).

Corresponding author: N.-S. Lin; E-mail: nslin@sinica.edu.tw; Telephone: +886-2-2787-1128 (office) +886-2-2787-1022 (lab); Fax: +886-2-27880991.

* The $\boldsymbol{e}$-Xtra logo stands for "electronic extra" and indicates that five supplementary figures and two supplementary tables are published online.

(C) 2014 The American Phytopathological Society
The key hormone in the response to abiotic stress is abscisic acid (ABA). ABA strongly antagonizes many hormone pathways, including SA (Soosaar et al. 2005; Yasuda et al. 2008), Et (Cheng et al. 2009; Ghassemian et al. 2000), and the synergistic Et/JA pathway (Broekaert et al. 2006). The role of ABA in plant defense was shown to be phase specific: ABA plays positive roles during the early stages of infection (for example, by inducing stomatal closure and callose deposition), and negative roles at later stages (which include suppressing ROS accumulation and SA induction) (Asselbergh et al. 2008; Munemasa et al. 2007; Ton et al. 2009).

Although the interactions between $\mathrm{ABA}$ and bacterial or fungal pathogens have been thoroughly studied, ABA-virus interactions are weakly addressed. Several studies showed that ABA is induced upon several viral infections but only a few works focused on how ABA is involved in plant defense to viruses. The earliest studies on ABA-virus interactions investigated the effect of Tobacco mosaic virus (TMV) infection on the accumulation of ABA in Nicotiana tabacum and tomato (Fraser and Whenham 1989; Whenham et al. 1986). ABA was found to mediate plant defense through increasing callose deposition and thereby limiting virus movement. Conversely, ABA content was not affected in potato cultivars infected with Potato virus $Y$ (Kovac et al. 2009). Tomato plants harboring Tm-1, which confers resistance to TMV, exhibited elevated levels of ABA as compared with susceptible lines (Fraser and Whenham 1989). Furthermore, whereas ABA is not induced in response to White clover mosaic virus infection in Phaseolus vulgaris (Clarke et al. 1998), it has a crucial role in mediating callose deposition and restricting movement of Tobacco necrosis virus (TNV) in the same host; these latter effects were not evident in $P$. vulgaris treated with an ABA inhibitor (Iriti and Faoro 2008). Banana infected with Banana bunchy top virus exhibited significant accumulation of ABA at 35 days postinoculation (dpi) (Zhang et al. 1997), and ABA levels were also increased in nonheading Chinese cabbage infected with Turnip mosaic virus (Wang et al. 2011). Infection with several RNA viruses, including Cucumber mosaic virus (CMV), Brome mosaic virus, and Tobacco rattle virus (TRV), confers improved host tolerance to drought, which is possibly a consequence of an increase in osmoprotectants and antioxidants (Xu et al. 2008). Despite the above observations, the role of ABA in plant defense against viruses requires further investigation.

Bamboo mosaic virus (BaMV) is a member of the Potexvirus genus, with a single-strand positive-sense RNA genome and a flexuous rod shape (Lin et al. 1994). The BaMV genome encodes five open reading frames (ORF) and produces genomic RNA (gRNA) (6.4 kb) and two major subgenomic RNAs 
(sgRNA) (2.0 and $1.0 \mathrm{~kb}$ ) during infection (Lin et al. 1992, 1994; Yang et al. 1997). BaMV moves systemically in N. benthamiana, in which it accumulates to high titers. It can also infect Arabidopsis thaliana but it does not undergo systemic movement; as such, it is present at lower titers and causes no visible symptoms (Lin et al. 2010).

Here, we investigate the requirement of the ABA pathway for BaMV accumulation in A. thaliana and N. benthamiana. Interestingly, BaMV titers were decreased in the ABA biosynthesis mutant aba2-1, and were affected to a lesser extent in a mutant of the upstream gene NCED3. However, plants with mutations in genes encoding proteins downstream of $A B A 2$ were more susceptible to infection. Thus, $A B A 2$ divides the ABA pathway into upstream genes required for BaMV accumulation and downstream genes required for plant resistance. These results were confirmed in $N$. benthamiana. We also report that infection with BaMV or CMV simultaneously induces the antagonistic ABA and SA pathways and results in significant accumulation of ABA. To our knowledge, this is the first study to describe the role of the ABA pathway in virus accumulation, thus uncovering a novel role for $A B A 2$ in mediating the accumulation of two different RNA viruses.

\section{RESULTS}

\section{BaMV accumulation is differentially affected in mutants of ABA biosynthesis.}

We first examined whether mutations of ABA biosynthesis affect the accumulation of BaMV in A. thaliana. To this end, we used mutants of the following genes: NCED3, $A B A 2$, and $A A O 3$. NCED3 encodes a member of the 9-cis-epoxycarotenoid dioxygenase family, responsible for converting 9-cisviolaxanthin and 9-cis-neoxanthin into xanthoxin (Schwartz et al. 2003). The nced3 mutant exhibits approximately $40 \%$ less ABA compared with wild-type (WT) plants, and fails to induce sufficient ABA under drought stress (Hwang et al. 2010; Wan and $\mathrm{Li}$ 2006). ABA2 encodes a short-chain alcohol dehydrogenase, which catalyzes the conversion of xanthoxin into ABAaldehyde; the aba2-1 mutant contains as little as approximately $22 \%$ of the ABA content of the WT (Gonzalez-Guzman et al. 2002). AAO3 catalyzes the conversion of ABA-aldehyde to ABA (Seo et al. 2000) and the aao3 mutant exhibits approximately $45 \%$ of the ABA content of the WT (Seo et al. 2004). BaMV infection of these three mutants yielded surprising results: whereas BaMV titers were significantly reduced in nced 3 and aba2-1 as compared with the WT (to approximately 60 and $25 \%$, respectively), they were increased in aao3 (approximately $400 \%$ ) compared with the WT (Fig. 1A).

To determine whether the reduction in viral titer in the necd 3 mutant was a consequence of its decreased ABA content, we used an enzymatic inhibitor for the NCED family, named nordihydroguaiaretic acid (NDGA). NDGA is known to reduce the enzymatic activity of NCEDs to approximately $50 \%$ as compared with the mock line, thereby reducing ABA content greatly and leading to stomatal closure (Creelman et al. 1992; Han et al. 2004; Zhang et al. 2009). Low concentrations of NDGA $(<1 \mathrm{mM})$ weakly reduced BaMV titers (data not shown) but higher concentrations $(10,20$, and $30 \mathrm{mM}$ ) clearly suppressed the accumulation of BaMV (Supplementary Fig. S1).

We also confirmed the role of ABA by applying it exogenously to leaves. NDGA and ABA were dissolved in $0.2 \%$ ethanol to concentrations of $10 \mathrm{mM}$ and $100 \mu \mathrm{M}$, respectively. Plants sprayed with $0.2 \%$ ethanol mock solution are hereafter referred to as $\mathrm{EtOH}$ lines. As expected, $10 \mathrm{mM}$ NDGA reduced BaMV titers to approximately $45 \%$. Interestingly, ABA also significantly reduced BaMV titers (Fig. 1B). To confirm that ex- ogenous $\mathrm{ABA}$ induced a response, we used quantitative reversetranscriptase polymerase chain reaction (qRT-PCR) to measure the levels of ABA-responsive genes $(A B A 2$ and $R A B 18)$ in treated plants. $A B A 2$ and $R A B 18$ were significantly upregulated (by two- and 44-fold, respectively) in the mock (not inoculated) ABA-treated plants compared with the control (Fig. 1C and D). This increase was more evident in BaMV-infected plants, with a 4-fold increase in $A B A 2$ and an approximately 27-fold increase in $R A B 18$ in ABA-treated plants compared with the EtOHinfected plants (Fig. 1C and D). Therefore, exogenous ABA application induced expression of ABA-responsive genes.

$A B A 2$ was significantly suppressed in BaMV-inoculated plants treated with NDGA, as compared with both EtOH- and ABA-treated lines (Fig. 1C). RAB18 levels were generally unaffected by NDGA; levels were the same as those in EtOHmock plants but significantly lower compared with those in the EtOH-infected line (Fig. 1D). We hypothesized that the level of $A B A 2$ may be affected in nced3, based on the reduced accumulation of BaMV in this mutant. Indeed, $A B A 2$ transcripts were significantly lower in infected nced3 lines than in the WT control, which justifies the low level of BaMV in nced3 (Supplementary Fig. S2).

\section{Mutations in the ABA signaling pathway increase BaMV levels.}

Based on the increased BaMV titers in aao3 (Fig. 1A), we hypothesized that BaMV accumulation may be similarly affected by mutants in other downstream components of this signaling pathway. We tested this hypothesis using the following ABA-insensitive mutants: abil-1, a gain-of-function dominant mutant of the negative regulator ABII (Leung et al. 1997), and $a b i 3-1$ and abi4-1, loss-of-function mutants of the positive regulators $A B I 3$ and $A B I 4$, respectively (Finkelstein 1994; Koornneef et al. 1984). Levels of BaMV RNA were two- to threefold higher than the WT in all ABA-insensitive mutants examined (Fig. 2A). Enzyme-linked immunosorbent assay (ELISA) revealed that ABA was increased in abil-1 and abi41 but not abi3-1 compared with their respective controls (mock Col-O for abi4-1 and Ler WT for abil-1 and abi3-1). BaMV infection resulted in a slight (and insignificant) increase in ABA in abi3-1 (Fig. 2B). This suggests that disrupting the ABA signaling pathway enhances susceptibility to BaMV, and this is independent of the levels of background ABA.

We proceeded to investigate whether the increase in ABA or BaMV levels correlated with increased expression of ABA biosynthesis genes. Levels of $A B A 2$ and $A A O 3$ were significantly increased in mock and infected abi4-1 lines as compared with Col-WT (Fig. 3B and C) but NCED3 was induced only in the mock line (Fig. 3A). Ler-WT and Col-WT exhibited similar changes in gene expression in response to BaMV infection. Expression of $N C E D 3$ was increased in mock and infected abil-1 but decreased in infected $a b i-3$ (Fig. 3E). The level of $A B A 2$ was significantly increased only in infected abil-1 (Fig. $3 \mathrm{~F}) . A A O 3$ was upregulated in both mock and infected $a b i-1$ and $a b i-3$ (Fig. 3G). Because ABA signaling was impaired in these lines, $R A B 18$ expression was reduced in both mock and infected plants (Fig. 3D and H). Taken together, these results indicate that disrupting the ABA signaling pathway downstream of $A B A 2$ enhances susceptibility to BaMV; moreover, these data suggest that the ABA pathway may at least partially be involved in the defense against $\mathrm{BaMV}$, and this may involve transcriptional regulation by $A B I 3$ and $A B I 4$.

\section{$A B A 2$ affects the accumulation of BaMV at different stages of replication.}

Of the examined mutants of ABA biosynthesis and signaling, only aba2-1 and, to a lesser extent, nced 3 exhibited reduced 
accumulation of BaMV. To further elucidate the role of $A B A 2$, we examined BaMV replication in aba2-1 and a transgenic line that constitutively overexpresses $A B A 2(A B A 2-O / E)$; ABA levels in this line are more than double that of the WT (Hwang et al. 2010; Lin et al. 2007). BaMV positive-strand (+)RNA was reduced 5-fold in $a b a 2-1$ and increased 0.5 -fold in the $A B A 2-O / E$ line compared with the WT (Fig. 4A). Similarly, negative-strand (-)RNA was reduced 20-fold in aba2-1 as compared with the WT but was unaffected in $A B A 2-O / E$ (Fig. 4B). Coat protein (CP) levels were significantly affected by the
$A B A 2$ level in a similar manner (Fig. 4C). The dramatic effect of aba2-1 on the accumulation of (-)RNA BaMV implies that ABA may affect the early stages of replication. In addition, we observed similar effects on BaMV accumulation in ABA line protoplasts (data not shown), suggesting that the effect of aba2-1 is exerted at the cellular level and not through cell-tocell movement.

We subsequently measured the expression levels of NCED3, $A B A 2, A A O 3$, and $R A B 18$ in these lines. NCED3 was significantly reduced in the $a b a 2-1$ mock and infected lines; it was
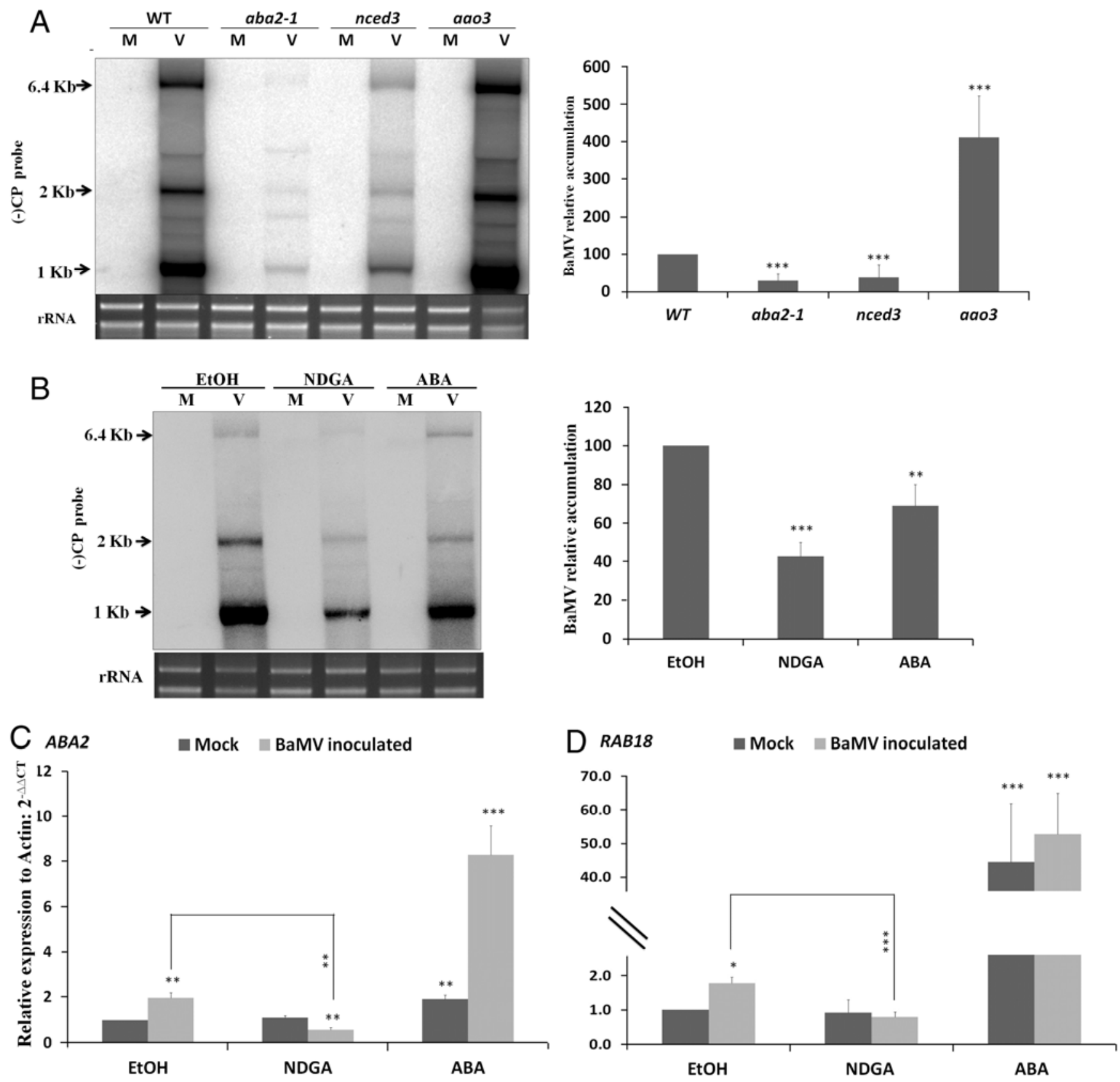

Fig. 1. Accumulation of BaMV is affected by abscisic acid (ABA) treatment and mutants of ABA biosynthesis. Northern blots of positive-strand BaMV genomic RNA and subgenomic RNAs in A, ABA biosynthesis mutants (nced3, aba2-1, or aao3) or B, wild-type (WT) plants treated with mock $(0.2 \%$ ethanol in $\mathrm{H}_{2} \mathrm{O}$ ), $10 \mathrm{mM}$ nordihydroguaiaretic acid (NDGA), or $100 \mu \mathrm{M}$ ABA. Plants were sprayed with the indicated chemical $12 \mathrm{~h}$ before inoculation with BaMV virions. Leaves were collected for RNA extraction and further processing at 10 days postinoculation (dpi) for the mutant lines and 6 dpi in the chemical treatment lines; $2 \mu \mathrm{g}$ of total RNA per sample was used to run the Northern blot. $\mathrm{M}=$ mock and $\mathrm{V}=\mathrm{BaMV}$ infected. Bar charts displaying the accumulation of $\mathrm{BaMV}$ genomic RNAs are shown on the right. Panels below the Northern blots indicate equal loading of total ribosomal RNA. Expression of $\mathbf{C}, A B A 2$ and D, $R A B 18$ in plants treated with $0.2 \%$ ethanol (mock), NDGA, or ABA, as determined using quantitative reverse-transcriptase polymerase chain reaction. Data are the mean \pm standard deviation from three biological replicates. One-sided $t$ test was performed to determine the significance of regulation. Asterisks indicate the significant differences by comparing the values of treatments to that of the uninfected WT or mock lines; $*, * *$, and $* * *$ represent $P<0.05,0.01$, and 0.005 , respectively. 
also reduced in the $A B A 2-O / E$ mock line but increased to WT levels upon viral infection (Fig. 4D). Interestingly, $A B A 2$ was not induced by BaMV infection in WT plants but was significantly reduced in the mock and infected $a b a 2-1$ mutant, as expected. The $A B A 2-O / E$ line exhibited tremendous upregulation of $A B A 2$, with approximately 100- and 70-fold increases in mock and infected lines, respectively, as compared with the WT (Fig. 4E). AAO3 expression was greater in mock $A B A 2-O / E$ than the WT, and increased significantly in all lines upon viral infection (Fig. 4F). RAB18 expression was also greater in mock
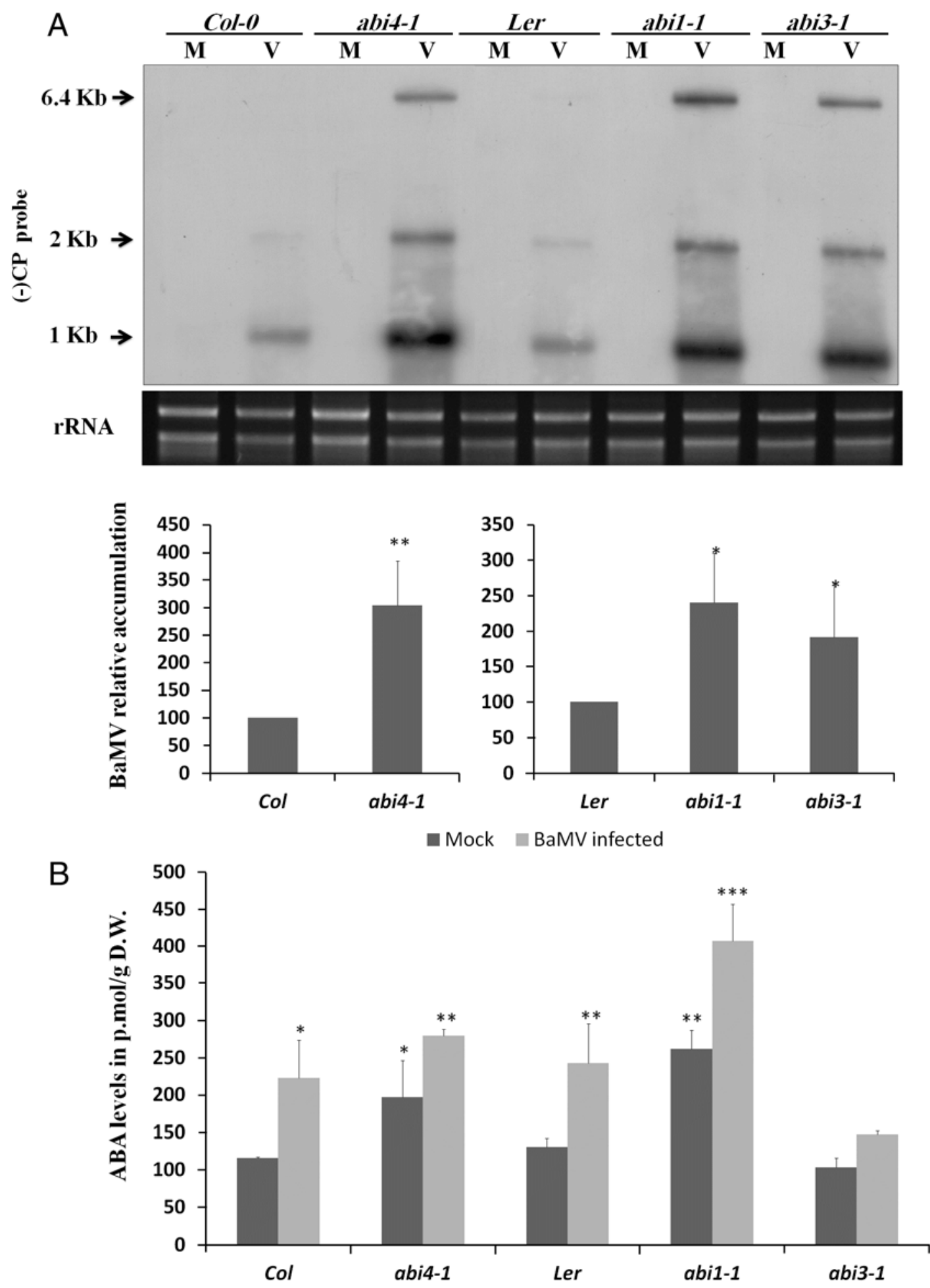

Fig. 2. BaMV accumulation and abscisic acid (ABA) content in ABA signaling mutants. A, Levels of BaMV in the ABA-insensitive mutant abi-4 as compared with its wild-type (WT) background Col-0, and in $a b i-1$ and $a b i-3$ as compared with WT Ler. Leaves were collected at 10 days postinoculation (dpi), and $2 \mu \mathrm{g}$ of total RNA was used for Northern blot. $\mathrm{M}=$ mock and V = BaMV infected. Lower panels indicate the levels of BaMV subgenomic RNA2. B, ABA content in mock or BaMV-infected abi mutants, as determined by enzyme-linked immunosorbent assay. Leaves were collected at 10 dpi for lyophilization and ABA extraction. ABA levels were measured in pmol per gram dry weight. One-sided $t$ test was performed to determine the significance of regulation. Asterisks indicate the significant differences by comparing the values of treatments to that of mock WT lines; *, **, and *** represent $P<0.05,0.01$, and 0.005 , respectively. Data are the mean \pm standard deviation from three biological replicates. Data are the mean \pm standard deviation from three biological replicates. 
$A B A 2-O / E$ compared with the WT, and increased in the WT following viral infection; conversely, no change was observed in the aba2-1 mutant (Fig. 4G).

Thus, $A B A 2$ and ABA seem to play different roles in plant defense. $A B A 2$ supports BaMV accumulation whereas ABA supports plant defense. To confirm this finding, we sprayed ABA onto the aba2-1 mutant. We predicted that the absence of $A B A 2$ would enhance the effect of ABA on plant defense. As expected, ABA treatment enhanced the resistance of aba2-1 to BaMV, because the viral titer was reduced to the lowest detectable level (Fig. 5). These findings support the conclusions that $A B A 2$ is required for $\mathrm{BaMV}$ to replicate but the hormone $\mathrm{ABA}$ is important for plant defense against BaMV infection.

\section{$A B A 2$ is important for CMV accumulation.}

We next asked whether $A B A 2$ had similar effects upon infection with other viruses. We chose to use CMV, because it suppresses SA-mediated defense and antiviral small interfering RNA machinery through its $2 \mathrm{~b}$ suppressor (Diaz-Pendon et al. 2007; Soosaar et al. 2005; Zhang et al. 2006). Surprisingly, the level of the CMV CP was reduced in aba2-1 compared with the WT and was not increased in $A B A 2-O / E$ (Fig. 6). Thus, the effect of aba2-1 on BaMV and CMV accumulation may be similar, and $A B A 2$ is required to support the accumulation of both viruses.

Virus-induced gene silencing knockdown of EDS1 and $A B A 2$ in $N$. benthamiana has effects on viral infection similar to those of the $A$. thaliana mutants eds 1-2 and aba2-1.

BaMV does not move systemically in A. thaliana; consequently, accumulation of this virus is approximately 10 -fold less in A. thaliana than in $N$. benthamiana, and it does not cause symptoms in $A$. thaliana (Lin et al. 2010). Furthermore, many RNA viruses, including BaMV, preferentially infect $N$. benthamiana (Goodin et al. 2008). Therefore, we examined whether $N$. benthamiana responded to BaMV infection in a similar manner as A. thaliana. To this end, we used virusinduced gene silencing (VIGS) to knockdown $N b A B A 2$. NbEDS1 was knocked down as a control because BaMV levels in the Arabidopsis SA mutant eds1-2 were found to be increased as compared with WT plants (Supplementary Fig. S4).

In the National Center for Biotechnology Information database, the approximately 650-bp sequence available for xanthoxin dehydrogenase (ABA2) of $N$. benthamiana (accession number EH371197) was blasted to the $N$. benthamiana expressed sequence tag (EST) database. The bigger EST of approximately 1,150 bp was blasted against Lycopersicum esculentum and $A$. thaliana $A B A 2$ (accession numbers XM 004237780 and NM_104113, respectively). NbABA2 showed approximately 82 and $69 \%$ identity with L. esculentum and $A$. thaliana, respectively (Supplementary Fig. S5; data not shown). Therefore, we used this sequence to choose a fragment for NbABA2 knockdown.

$N$. benthamiana plants were agroinfiltrated with Agrobacterium tumefaciens harboring the binary vectors pTRV1 and pTRV2; the latter had a small insert of approximately 250 to 500 bp (either $N b E D S 1$ or $N b A B A 2$, respectively). At 7 days postinfiltration, three leaves were inoculated with $0.2 \mu \mathrm{g}$ of BaMV RNA each, and inoculated leaves from two to three plants were collected at 7 dpi for RNA extraction. Although knocking down EDS1 enhanced BaMV titers as compared with the empty vector ( $\mathrm{pEV}$ ), silencing $N b A B A 2$ significantly decreased BaMV accumulation (Fig. 7A and D). VIGS reduced the transcript levels of $A B A 2$ and EDS1-2 to approximately $40 \%$ of that of plants infiltrated with pEV (Fig. 7B and E), confirming the efficacy of knockdown. Therefore, decreases in $A B A 2$ had similar effects on BaMV infection in Arabidopsis thaliana and $N$. benthamiana (Figs. 1 and 7). We also took advantage of previous characterization of $A B A 1$ (a few earlier works termed it as $A B A 2$ in N. tabacum and N. plumbaginifolia, although it encodes zeaxanthin epoxidase, whose ortholog in Arabidopsis is ABA1) (Audran et al. 1998; Marin et al. 1996) and knocked down this transcript by agroinfiltrating pTRV2 carrying approximately $250 \mathrm{bp}$ of $N b A B A 1$. Interestingly, knocking down $N b A B A 1$ also reduced BaMV titer compared with $p E V$-infiltrated plants (Fig. 7A). The transcript level of $N b A B A 1$ was reduced to $40 \%$ compared with the control plants (Fig. 7B). To investigate whether the levels of $N b A B A 2$
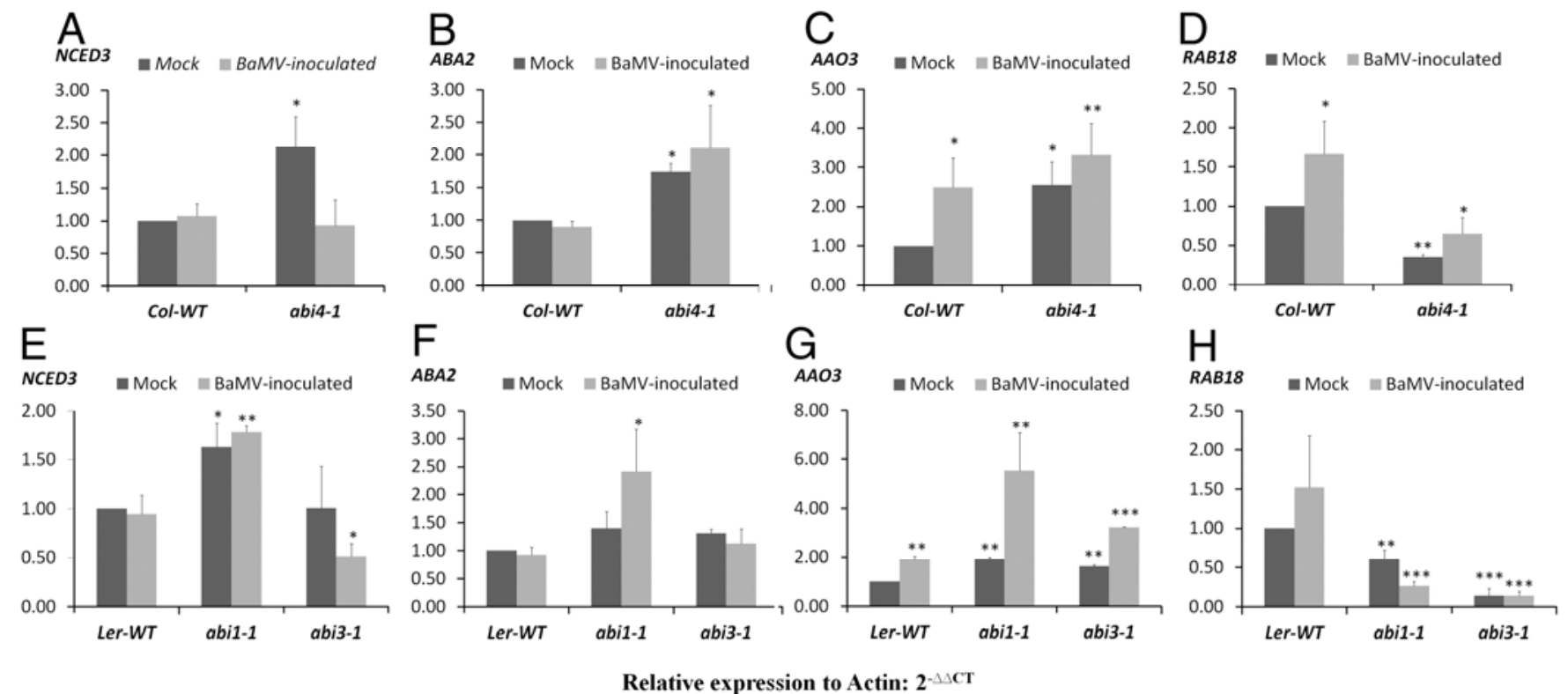

Relative expression to Actin: 2- $\triangle \triangle C T$

Fig. 3. Expression of abscisic acid (ABA) biosynthesis genes in $a b i$-insensitive mutants following BaMV infection. Transcripts levels of A and E, NCED3; B and $\mathbf{F}, A B A 2$; $\mathbf{C}$ and $\mathbf{G}, A A O 3$; and $\mathbf{D}$ and $\mathbf{H}, R A B 18$ in $\mathbf{A}$ to $\mathbf{D}, a b i-4$ compared with its Col wild-type (WT) background and $\mathbf{F}$ to $\mathbf{H}, a b i-1$ and $a b i-3$ compared with their Ler WT background. Data are the mean \pm standard deviation from three biological replicates. Data were normalized to the expression of each gene in mock-inoculated WT. One-sided $t$ test was performed to determine the significance of regulation. Asterisks indicate the significant differences by comparing the values of treatments to that of the mock WT lines; *, **, and *** represent $P<0.05,0.01$, and 0.005 , respectively. 

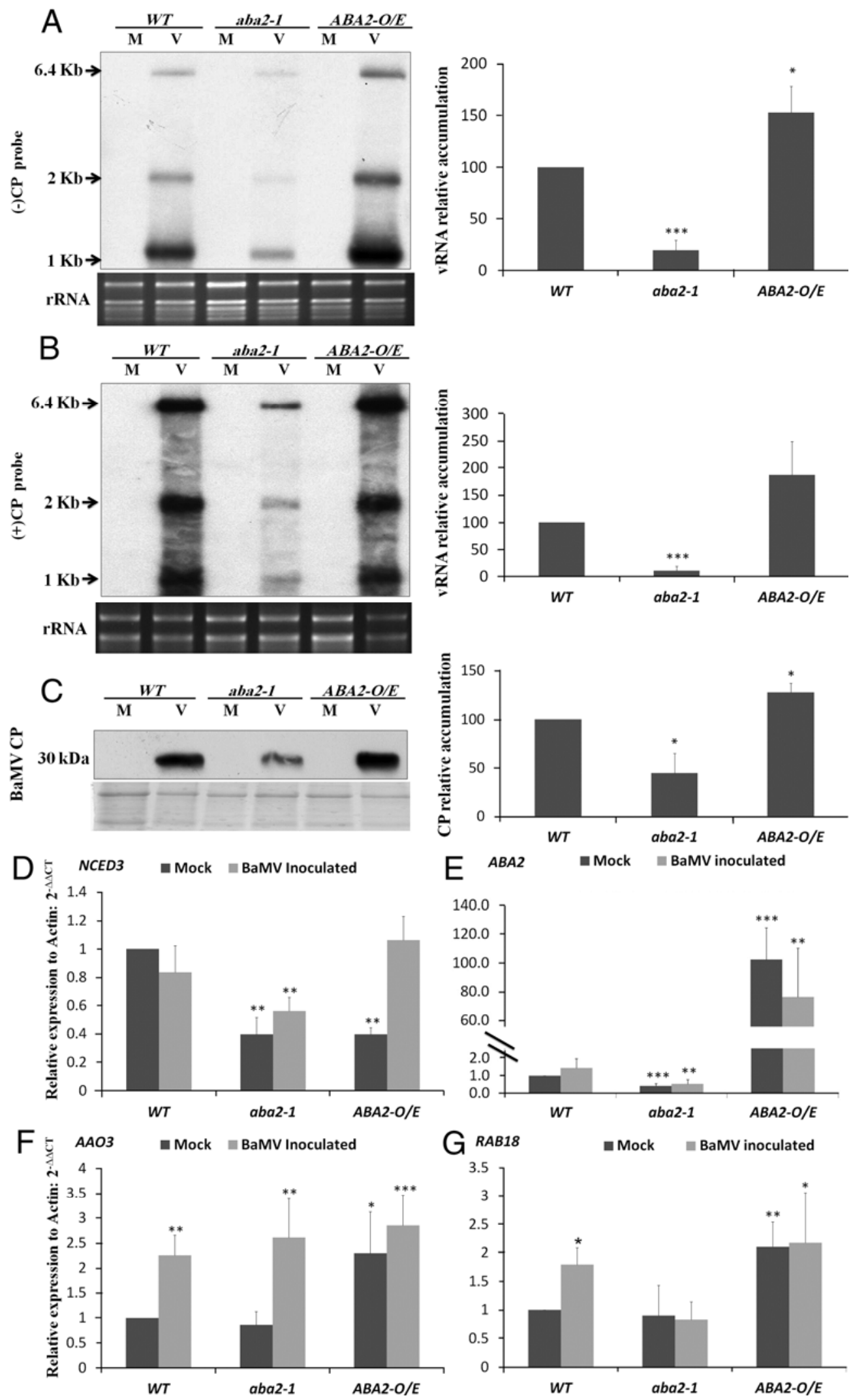

Fig. 4. Accumulation of BaMV and abscisic acid (ABA) genes in ABA lines. Accumulation of A, BaMV positive-strand RNA; B, negative-strand RNA; and C, coat protein (CP) in wild-type (WT), aba2-1, and $A B A 2$-overexpressing transgenic line $(A B A 2-O / E)$. Bar charts showing comparative accumulation are shown on the right. Total RNA and protein were extracted at 10 days postinoculation (dpi) from inoculated leaves, and $2 \mu \mathrm{g}$ of total RNA was used for Northern blot. C, Approximately $25 \mu \mathrm{g}$ of total protein were used for sodium dodecyl sulfate-polyacrylamide gel electrophoresis; membranes were hybridized with BaMV anti-CP sera. Lower panels show Coomassie blue staining as a loading control. Expression levels of D, NCED3; E, ABA2; F, AAO3; and G, $R A B 18$ in WT, aba2-1, and $A B A 2-O / E$ lines, as determined using quantitative reverse-transcriptase polymerase chain reaction. Data are the mean \pm standard deviation from three biological replicates. Data were normalized to the expression of each gene in mock-uninoculated WT. One-sided $t$ test was performed to determine the significance of regulation. Asterisks indicate the significant differences by comparing the values of treatments to that of the mock WT lines; $*, * *$, and $* * *$ represent $P<0.05,0.01$, and 0.005 , respectively. 
are related to the low BaMV titer in the $A B A 1$-knockdown line, we used qRT-PCR to measure $N b A B A 2$. We found that $N b A B A 2$ was also reduced approximately $48 \%$ in plants knocked down with $N b A B A l$ (Fig. 7C). This strengthens the finding that BaMV titers are positively correlated with the levels of $A B A 2$. Therefore, we used $N$. benthamiana for further experiments.

\section{CMV and BaMV infection increase ABA content and} activate the ABA and SA pathways in $N$. benthamiana.

ABA accumulation was greater in both CMV- and BaMVinfected $N$. benthamiana than in mock plants (Fig. 8A). Furthermore, mRNA levels of all tested ABA genes ( $A B A 1, A B A 2$, $A A O 3, A B I 3, A B I 5$, and $R A B 18)$ as well as SA genes (EDS1, SID2, NPR 1, PR-1, PR-4, and PR-5) were increased in CMVinfected $N$. benthamiana as compared with the WT (Fig. 8B, left panel; Supplementary Table S1). All examined SA and ABA genes were also upregulated in BaMV-infected plants but not $A B A 1$ (Fig. 8B, right panel). However, upregulation of the affected genes was greater in CMV-infected than in BaMVinfected plants. Therefore, CMV and BaMV infections both induce two antagonistic pathways: ABA and SA.

\section{DISCUSSION}

Previous studies have shown that exogenous application of ABA enhances the resistance of different plant hosts to viruses (such as TMV and TNV) by increasing callose deposition and thereby limiting viral movement (Fraser and Whenham 1989; Iriti and Faoro 2008; Whenham et al. 1986). We analyzed the effects of the ABA pathway on BaMV infection in depth by using ABA biosynthesis and signaling mutants and exogenous application of ABA and ABA inhibitor. Through this study, we identified a novel role for $A B A 2$ in supporting the accumulation of two different RNA viruses, BaMV and CMV. Conversely, we found that $A A O 3$ and its downstream genes are important for plant defense, indicating that ABA signaling is required for defense against viruses. Therefore, the effect of $A B A 2$ on $\mathrm{BaMV}$ is distinct from the ABA pathway itself.

ABA2 protein is encoded by a single gene in the Arabidopsis genome. It converts xanthoxin to ABA-aldehyde, which is used by $\mathrm{AAO} 3$ and the cofactor $\mathrm{ABA} 3$ to produce $\mathrm{ABA}$ (Hauser et al. 2011; Nambara and Marion-Poll 2005). The recognition of ABA by the PYR/PYL/RCAR receptor results in the dissociation of the SnRK2/PP2C complex and the subsequent release of SnRK2s. The latter phosphorylate and activate downstream transcription factors, which initiate transcription at ABA-responsive promoter elements (Sheard and Zheng 2009).

The ABA biosynthesis pathway mutants aba2-1 and, to a lesser extent, the upstream mutant nced3 result in decreased BaMV titers, whereas aao3 enhances viral titers (Fig. 1). All signaling pathway mutants responded similarly to aao3, by ex-
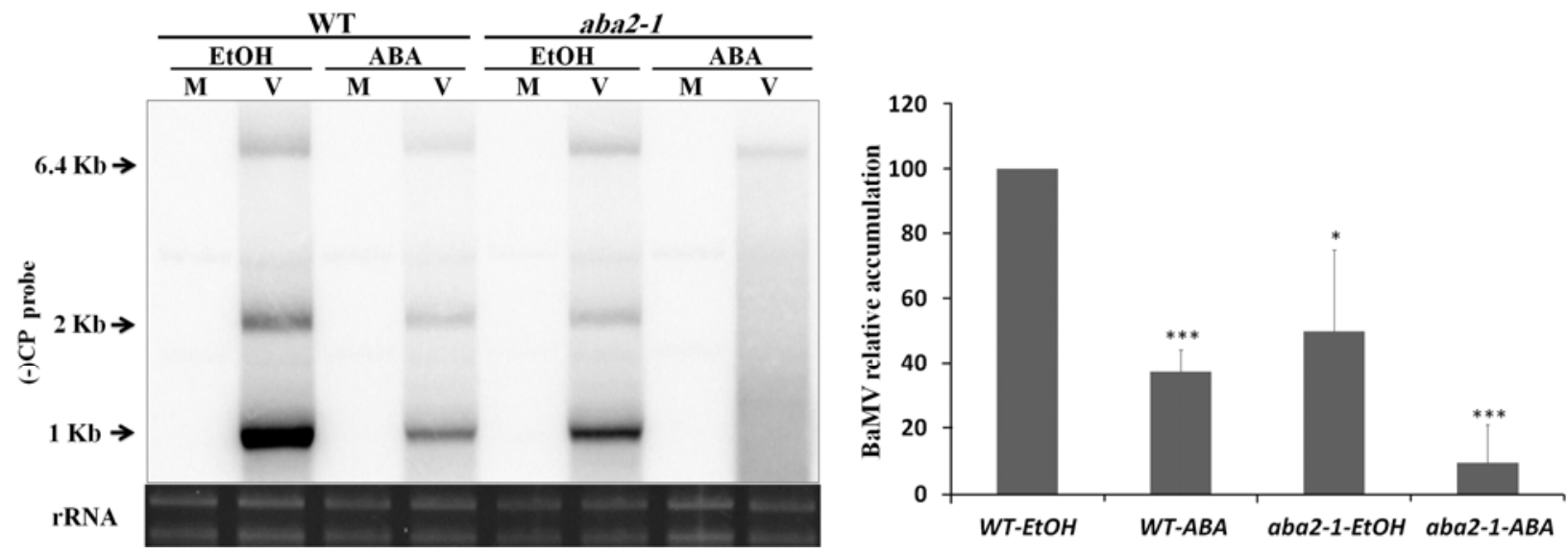

Fig. 5. Effect of exogenous application of abscisic acid (ABA) on BaMV accumulation in wild-type (WT) and aba2-1 plants. Northern blot analysis of positive-strand BaMV genomic RNA and two subgenomic RNAs in WT and aba2-1 plants. Plants were sprayed with ABA $12 \mathrm{~h}$ prior to BaMV infection, leaves were collected at 6 days postinoculation (dpi), and $2 \mu \mathrm{g}$ of total RNA was used for Northern blot. Data are the mean \pm standard deviation from three biological replicates. One-sided $t$ test was performed to determine the significance of regulation. Asterisks indicate the significant differences by comparing the values of treatments to that of the uninfected WT-EtOH line, * and *** represent $P<0.05$ and 0.005 , respectively.
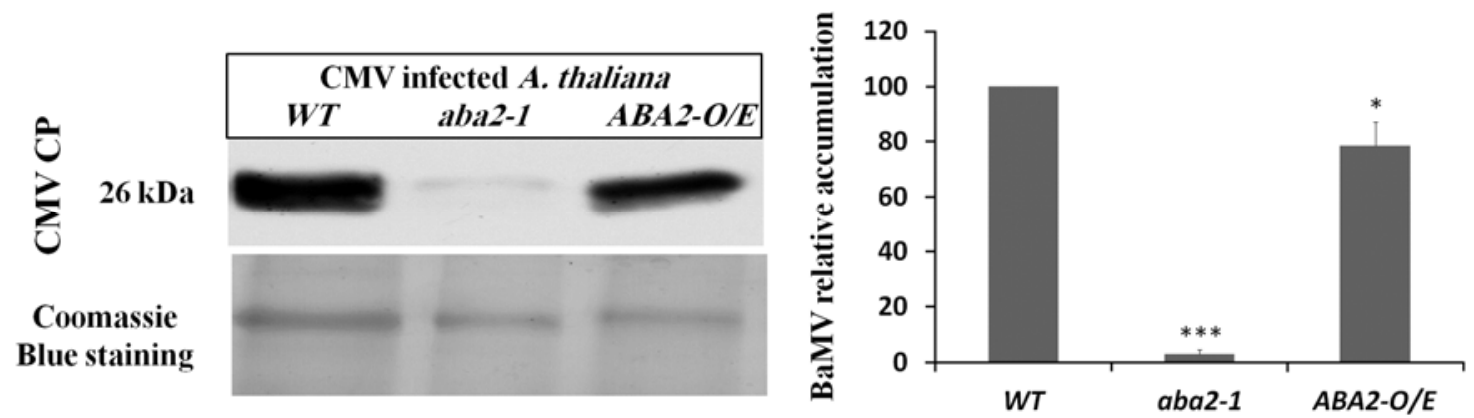

Fig. 6. CMV accumulation in the aba2-1 mutant. Arabidopsis thaliana lines were infected with crude extracts of CMV-infected Nicotiana benthamiana. At 10 days postinoculation (dpi), inoculated leaves were collected for protein extraction; approximately $25 \mu \mathrm{g}$ total protein were used for sodium dodecyl sulfatepolyacrylamide gel electrophoresis, and membranes were probed with anti-CMV coat protein (CP). The lower panel shows Coomassie blue staining as a loading control. The bar chart shows accumulation of CMV CP. Data are the mean \pm standard deviation from three biological replicates. One-sided $t$ test was performed to determine the significance of regulation. Asterisks indicate the significant differences by comparing the values of treatments to that of the WT line, * and *** represent $P<0.05$ and 0.005 , respectively. 
hibiting enhanced BaMV titers (Fig. 2). These results suggest that genes downstream of $A B A 2$ are important for plant resistance to BaMV. The higher viral titer in the aao3 mutant (which fails to encode the protein required for the final step of ABA synthesis) strongly suggests that ABA is important for plant defense. The following lines of evidence support this conclusion: i) exogenous application of ABA reduced the BaMV titer in WT plants (Fig. 5) and ii) viral titers were increased in signaling pathway mutants (abil-1, abi3-1, and abi4-1) in a similar manner as in aao3 (Fig. 2A). Although ABA was induced in BaMV-infected abil-1 and abi4-1 lines (Fig. 2B), disruption of the signaling pathway prevented ABA-mediated resistance to BaMV. The similar effects on viral accumulation observed in abil-1, abi3-1, and abi4-1 were not unexpected; abil-1 is a gain-of-function mutant of the negative regulator $\mathrm{ABI}$, in which the PP2C-SnRK2 complex is insensitive to the

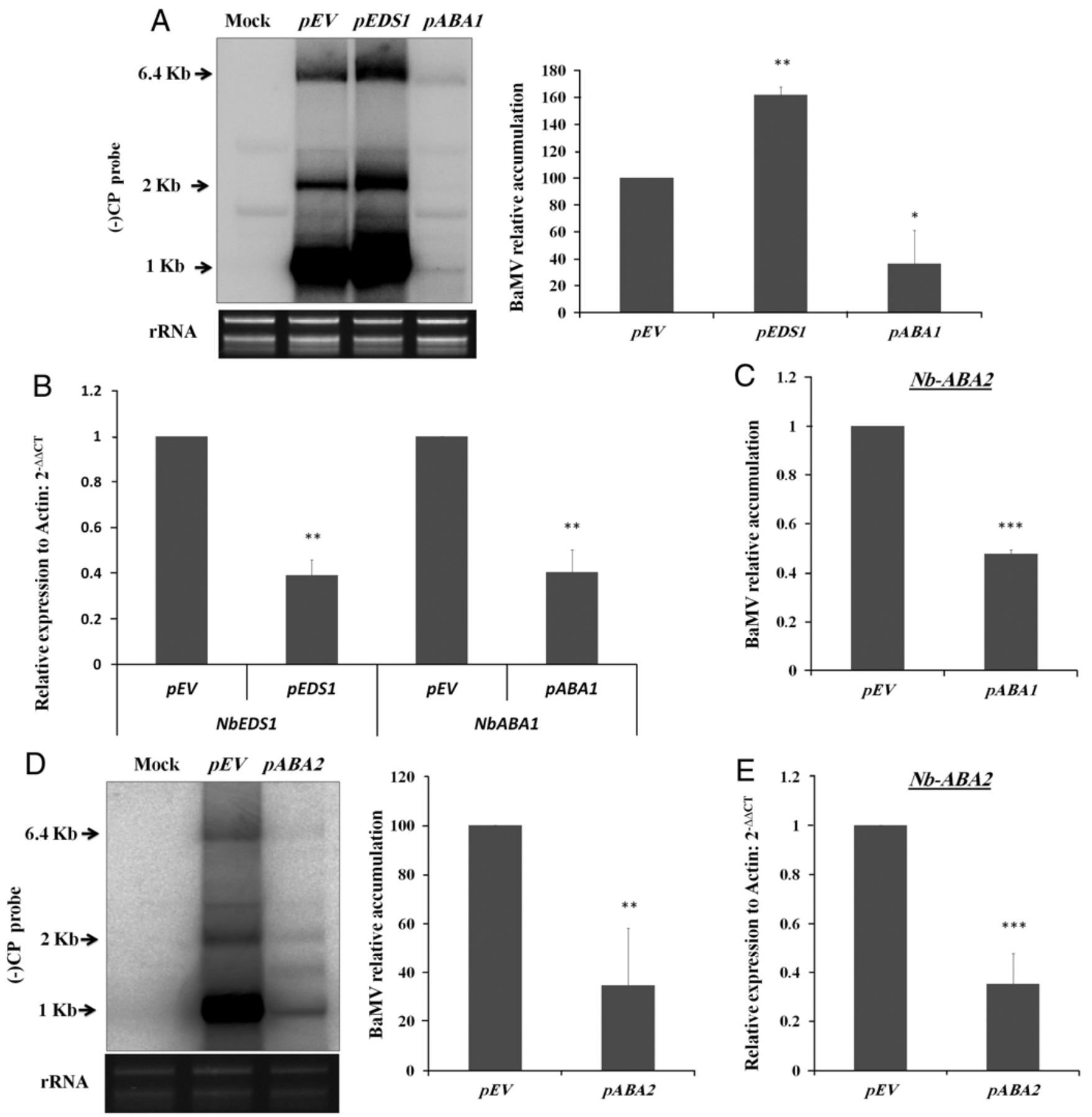

Fig. 7. Effect of virus-induced gene silencing (VIGS) on BaMV accumulation in Nicotiana benthamiana. A, BaMV accumulation after VIGS knockdown of $N b E D S 1$ and $N b A B A 1$ as revealed by Northern blot. Plants were infiltrated with $p E V(p T R V 1+p T R V 2), p E D S 1$ ( $p T R V 1+p T R V 2:: E D S 1)$, or $p A B A 1$ ( $p T R V 1$ + pTRV2::ABA2), and inoculated with $0.2 \mu \mathrm{g}$ of BaMV RNA at 7 days postinfiltration. Inoculated leaves were collected at 7 days postinoculation (dpi) for RNA extraction, and $2 \mu \mathrm{g}$ of total RNA was used for Northern blot. The bar chart shows the relative accumulation of BaMV in $N$. benthamiana. B, Relative expression of $N b E D S 1$ and $N b A B A 1$ in the indicated plants. C, Expression level of $N b A B A 2$ in plants knocked down with $N b A B A 1$ compared with pEV plants. D, BaMV accumulation after VIGS knockdown of NbABA2. The bar chart shows the relative accumulation of BaMV in $N$. benthamiana. E, Relative expression of $N b A B A 2$ in the $A B A 2$-silenced plants. Data are the mean \pm standard deviation from three biological replicates. Data were normalized to the expression of each gene in $\mathrm{pEV}$ line. One-sided $t$ test was performed to determine the significance of regulation. Asterisks indicate the significant differences by comparing the values of treatments to that of the uninfected $\mathrm{pEV}$ line. * and *** represent $P<0.05$ and 0.005 , respectively. 
ABA; thus, SnRK2 will not be released from the complex, nor will it phosphorylate or be able to activate ABA-responsive elements (Sheard and Zheng 2009). Therefore, no ABA-defense mechanism will be activated in this mutant. Similarly, abi3-1 and abi4-1 are loss-of-function mutants of the positive regulators $\mathrm{ABI} 3$ and $\mathrm{ABI} 4$, in which the ABA signaling pathway is impaired and results, likewise, in no activation of ABA-related defense. However, although ABA levels were increased in abil-1 and abi4-1 lines as compared with the WT, they were unaffected in abi3-1; this may be related to the observation that expression of NCED3 and $A B A 2$ were decreased and unaffected, respectively, in this line as compared with the WT (Fig. 3E and F). We hypothesize that ABA-mediated defense is possibly transcriptionally regulated by $A B I 3$ and $A B I 4$, because both encode transcription factors that regulate ABA-responsive genes (Finkelstein et al. 1998; Giraudat et al. 1992). Comparison of the aba2-1 mutant and an $A B A 2-O / E$ transgenic line clearly demonstrated the importance of $A B A 2$ for BaMV accumulation; aba2-1 negatively affects the accumulation of BaMV (+)RNA, $(-)$ RNA, and CP, with the effect on (-)RNA being most profound (Fig. 4). This implies that lack of ABA2 activity probably affects the early steps of BaMV replication (Fig. 9). We observed low titers of BaMV in lines with significantly reduced levels of $A B A 2$, such as NDGA-treated and nced3 lines (Fig. 1B). Although exogenous application of ABA significantly de- creased BaMV titers (Fig. 1B), ABA2 levels were also increased fourfold by viral infection (Fig. 1C). This may be why a clearcut effect of exogenous ABA was not observed. The effects of $\mathrm{ABA}$ and $A B A 2$ on plant defense were demonstrated by treating aba2-1 mutant plants with ABA, which reduced BaMV titers to almost undetectable levels (Fig. 5). This result also reveals that $A B A 2$ promotes BaMV accumulation in opposition to $\mathrm{ABA}$, suggesting that ABA and aba2-1 may affect different mechanisms. Knocking down $A B A 2$ in $N$. benthamiana had an effect on BaMV titers similar to that of the aba2-1 mutant (Fig. 7). In fact, a recent study showed that ABA is important for plant resistance to TMVcg infection. However, this study found that the aba2 mutant, like other downstream mutants, enhanced plant susceptibility to TMVcg infection, showing no distinct role of $A B A 2$ to other ABA mutants (Chen et al. 2013), contrary to our findings for BaMV and CMV. This strengthens the probability that ABA2 is involved in replication or translation complexes for $\mathrm{BaMV}$ and $\mathrm{CMV}$, and that such involvement is specific to some viruses and cannot be generalized. In fact, not all RNA viruses may respond similarly to the same mutant. Huang and associates (2005) found that CMV-Y and Oil seed mosaic virus were not affected by several SA mutants such as eds1, whereas BaMV accumulated more in this mutant.

Viral titers in the $A B A 2-O / E$ line were increased by $50 \%$ as compared with the WT (Fig. 4). In addition, expression of ABA

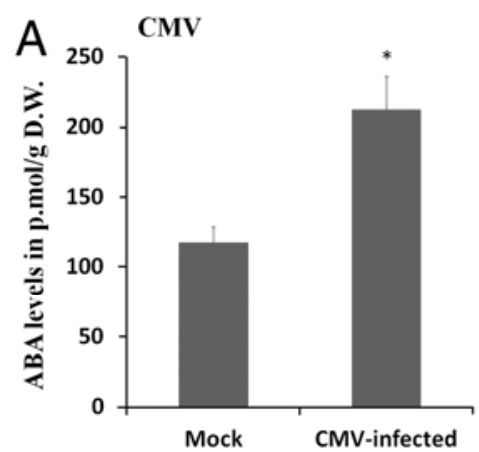

B

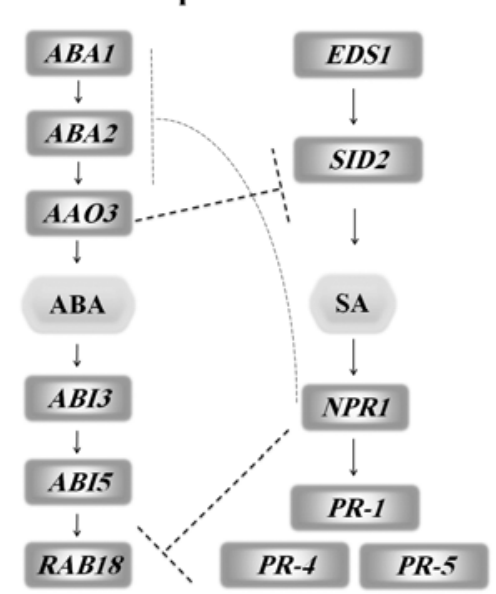

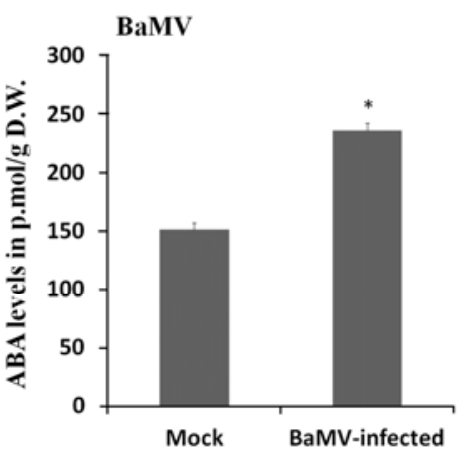

Response to BaMV

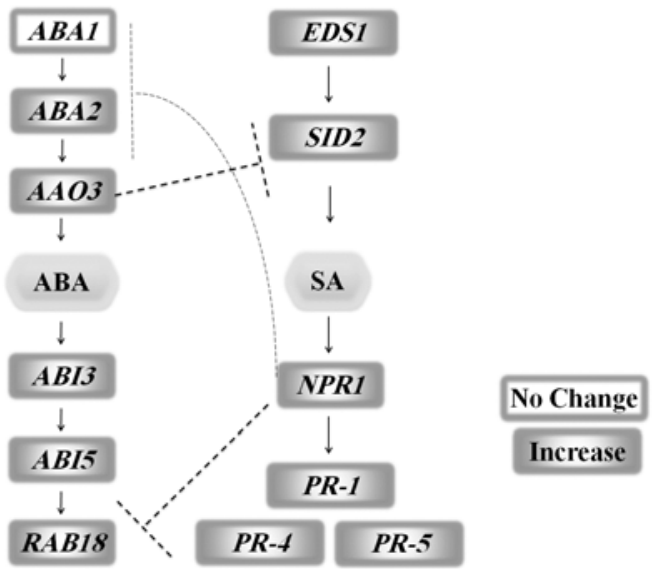

Fig. 8. Effect of CMV and BaMV infection on abscisic acid (ABA) content and defense gene expression in Nicotiana benthamiana. A, Effect of CMV (left panel) and BaMV (right panel) infection on ABA content in $N$. benthamiana. Systemic leaves of infected N. benthamiana were collected at 16 days postinoculation (dpi) and subjected to enzyme-linked immunosorbent assay to determine ABA content. ABA levels were measured in pmol per gram dry weight. One-sided $t$ test was performed to determine the significance of regulation. Asterisks indicate the significant differences by comparing the values of treatments to that of the mock line, * represents $P<0.05$. B, Changes in expression of the main defense pathway genes following CMV (left panel) or BaMV (right panel) infection at $16 \mathrm{dpi}$ were determined using quantitative reverse-transcriptase polymerase chain reaction. Dashed lines mean putative negative effect or blockage; NPR1 has a negative effect over ABA biosynthesis genes $A B A 1$ and $A B A 2$, as well as over some ABA-responsive genes such as $R A B 18$, while the aao3 mutant allowed more SID2 to accumulate (Yasuda et al. 2008). The upregulation of these transcripts implies that ABA-salicylic acid (SA) antagonism is disrupted by CMV or BaMV infection. Gray boxes indicate an increase of twofold or more and white boxes indicate no change. Values were normalized to actin. 
genes (with the exception of NCED3) was increased in this line (Fig. 4). $A B A 2-O / E$ seems to have a dual effect on BaMV and plant defense. Overexpression of $A B A 2$ supports $\mathrm{BaMV}$ accumulation and enhanced levels of ABA support plant resistance to the virus. These opposing effects account for the weak increase in BaMV titer in $A B A 2-O / E$ (Figs. 4 and 9). These findings strongly suggest that i) either certain defense mechanisms are negatively regulated by $\mathrm{ABA} 2$, thereby affecting early events of the replication cycle, or BaMV-related proteins are positively regulated by $A B A 2$ directly or indirectly and ii) the ABA signaling pathway partially underlies plant resistance to BaMV infection. Another possibility is that ABA2 may have indirect effects through its substrates; in the absence of functional ABA2, metabolic flux through this pathway is disrupted; this may lead to the accumulation of precursor metabolites or compounds derived from alternative pathways, which inhibit virus replication.

We also used CMV infection to demonstrate the importance of $A B A 2$ in $\mathrm{CP}$ accumulation. CMV CP levels were greatly reduced in the aba2-1 mutant but unaffected in the $A B A 2-O / E$ line (Fig. 6); the latter finding may be due to the conflicting effects of increased ABA2 and ABA. Thus, $A B A 2$ appears to be required for the accumulation of $\mathrm{CMV}$, in a manner similar to BaMV.

In $N$. benthamiana, infection with $\mathrm{CMV}$ or BaMV induced ABA accumulation (Fig. 8A) and upregulated the mRNA levels of several genes in the SA and ABA pathways (Fig. 8B). This finding suggests that the induction of $\mathrm{ABA}$ and upregulation of the ABA and SA pathways may be common features of RNA virus infection. The disruption of ABA-SA antagonism was shown by the upregulation of almost all tested genes in each pathway. In fact, Yasuda and associates (2008) showed that
$A A O 3$ has a suppressive effect on SID2, also NPR1 has a suppressive effect on different ABA biosynthesis and downstream genes such as $A B A 2$ and $R A B 18$ (Yasuda et al. 2008). Our data showed that all of these genes, along with the rest of the tested ABA and SA genes (six per each pathway), were upregulated simultaneously under CMV or BaMV infection regardless of their antagonistic effects on the opposite pathway (Fig. 8B). This result implies that infection with these two RNA viruses disrupts the ABA-SA antagonism. Supportively, a recent study showed that the $2 \mathrm{~b}$ silencing suppressor of CMV interferes with the ABA pathway and renders A. thaliana plants more tolerant to drought (Westwood et al. 2013). Another study reported that disruptions in ABA biosynthesis but not ABA signaling enhanced the nuclear localization of two resistance proteins, SNCl and RPS4, and negated the effect of these proteins on disease resistance at high temperature. We note that this work examined abal-6 and aba2-1 mutants but not the downstream mutant aao3 (Mang et al. 2012). The positive effect of the mutant aba2-1 on plant resistance to BaMV or CMV hypothesizes that there could be a defense mechanism which might be negatively regulated by $A B A 2$, because low levels of $A B A 2$ always lead to low BaMV or CMV titers. The other probability is that ABA2 could be an essential factor in the replication or translation complex so that, by reducing ABA2 levels, this affects the replication or translation efficiency and results in low-level viral replication forms, as shown for (+)RNA and (-)RNA of BaMV (Figs. 4 and 9). The nature of ABA2 as a dehydrogenase enzyme bears another probability, which is the associated NAD coenzyme (Gonzalez-Guzman et al. 2002) or other associated factors, and that by mutating or downregulating $A B A 2$, NAD coenzyme will be more abundant and can affect the redox state in the cell. The redox state was

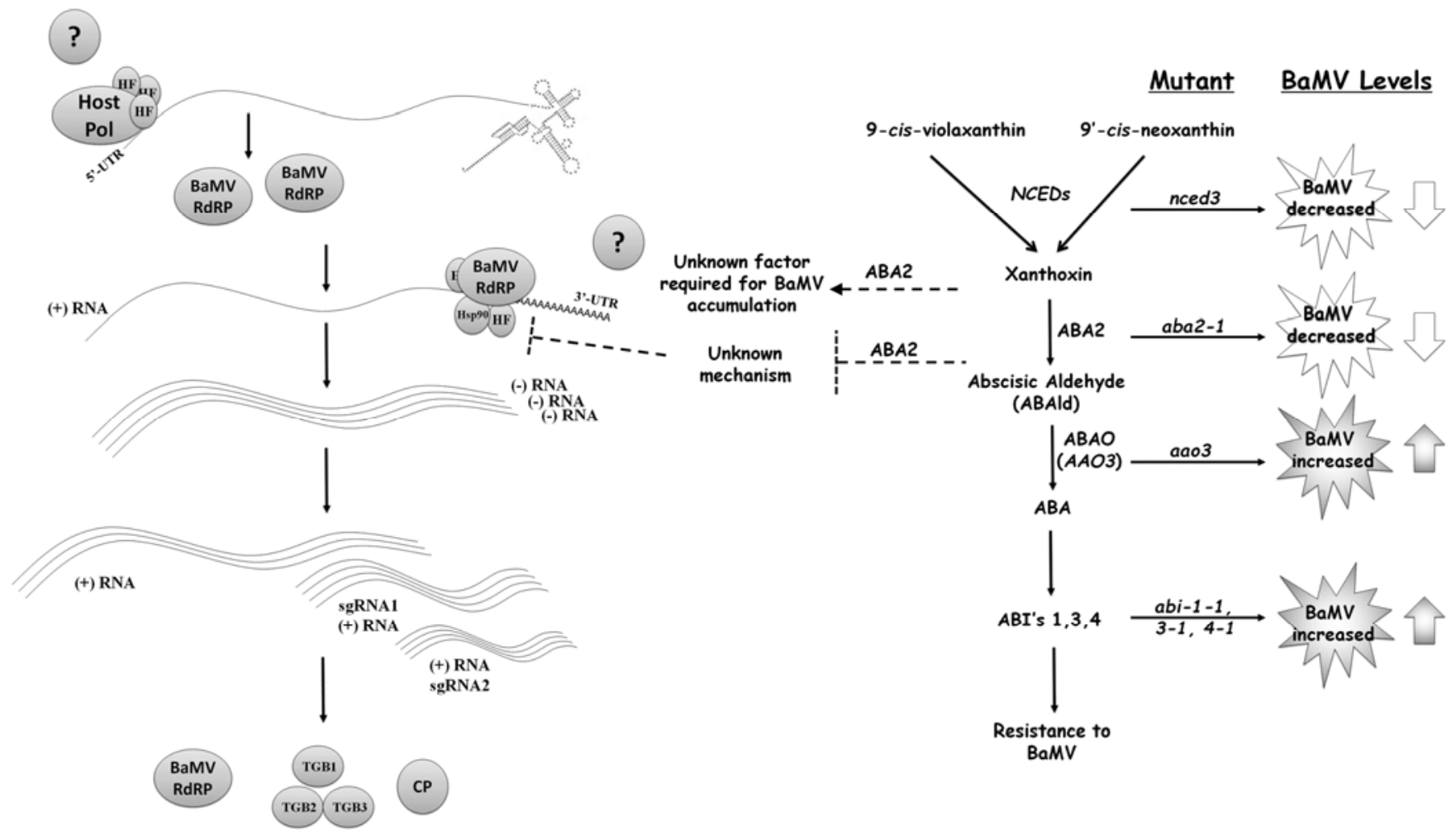

Fig. 9. Schematic representation of the effect of the abscisic acid (ABA) pathway on BaMV accumulation. The ABA pathway has two different effects on BaMV accumulation. Impairing ABA biosynthesis through the nced 3 and aba2-1 mutants reduces BaMV titers in plants; aba2-1 appears to affect BaMV replication primarily by decreasing (-)RNA and also by reducing (+)RNA and coat protein (CP) accumulation. Mutants downstream of aba2-1 (such as aao3) or mutants in the ABA signaling pathway (such as abil-1, abi3-1, and abi4-1) also increased BaMV titers, indicating that ABA pathway components downstream of $a b a 2-1$ are involved in the resistance against BaMV. $A B A 2$ appears to be required to stabilize and support BaMV replication by acting on certain required factors or by negatively affecting an anti-BaMV mechanism. 
found to be connected to plant basic immunity (Spoel and Dong 2012) and was also found to be involved in the regulation of RNA-protein bindings (Barnes and Mayfield 2003; Woloshen et al. 2011), which means there is a possibility of interference in the binding process between viral RNA and host proteins. Further studies on how ABA2 regulates BaMV replication are required to unveil any possible connection between ABA2 and defense systems against viruses.

To our knowledge, this is the first report to demonstrate that $A B A 2$ promotes accumulation of two different RNA viruses, while downstream genes in ABA biosynthesis and the ABA signaling pathway enhance plant resistance to BaMV. It was also found that infection with BaMV or CMV can simultaneously induce two antagonistic pathways, SA and ABA.

\section{MATERIALS AND METHODS}

\section{Plant materials and growth conditions.}

WT $N$. benthamiana plants were grown under long-day conditions at $28^{\circ} \mathrm{C}$ with a relative humidity of approximately $70 \%$. A. thaliana lines were grown under long-day conditions at $22^{\circ} \mathrm{C}$ with a relative humidity of approximately $60 \%$.

\section{Inoculation with BaMV.}

Purified BaMV RNA was extracted from virions as previously described (Lin et al. 1992). Three leaves of each A. thaliana or $N$. benthamiana plant (approximately six replicate plants per line) were inoculated with $0.1 \mu \mathrm{g}$ of BaMV RNA, unless otherwise stated. The inoculated leaves were collected from $A$. thaliana and $N$. benthamiana at 10 and $16 \mathrm{dpi}$, respectively, for further analyses.

\section{Treatment with ABA and ABA inhibitor.}

A. thaliana leaves were treated with $\mathrm{ABA}$ or an ABA biosynthesis inhibitor, NDGA, which targets 9-cis-epoxycarotenoid dioxygenase. Three concentrations of NDGA were prepared: 10,20 , and $30 \mathrm{mM}$. ABA was prepared at $100 \mu \mathrm{M}$ and all solutions were dissolved in $0.2 \%$ EtOH- $\mathrm{H}_{2} \mathrm{O}$. Mock controls were treated with $0.2 \%$ EtOH- $\mathrm{H}_{2} \mathrm{O}$ alone. All chemicals were from Sigma-Aldrich (St. Louis). Solutions were sprayed onto the adaxial and abaxial sides of leaves until they were completely coated (at which point, the solutions began to run off the leaves). After $12 \mathrm{~h}$, leaves were mechanically infected with $1 \mu \mathrm{g}$ of BaMV virions; at $6 \mathrm{dpi}$, RNA was extracted for use in subsequent analyses.

\section{RNA analysis.}

Total RNA was extracted from leaves by TRIzol (Invitrogen, Carlsbad, CA, U.S.A.), was purified using the phenol-chloroform method, and was precipitated in 0.1 volume of $3 \mathrm{M}$ $\mathrm{NaOAc}$ and 2.5 volume of $100 \%$ ethanol.

Northern blot analysis: BaMV RNA was detected as follows: total RNA $(2 \mu \mathrm{g})$ was glyoxylated and then separated by electrophoresis on a $1 \%$ agarose gel. The RNA was then transferred onto a Hybond- ${ }^{+}$membrane (GE Healthcare, Little Chalfont, Buckinghamshire, U.K.), cross-linked under UV light, and hybridized against $\mathrm{P}^{32}$-labeled $(-) \mathrm{CP}$ and $(+) \mathrm{CP}$ probes to detect (+)BaMV and (-)BaMV, respectively (Lin et al. 1993).

$q R T-P C R$. Each RNA sample $(2 \mu \mathrm{g})$ was treated with RQ1DNAse (Promega, Madison, WI, U.S.A.) for $30 \mathrm{~min}$ at $37^{\circ} \mathrm{C}$. RNA samples were then subjected to first-strand cDNA synthesis with Superscript III (Invitrogen). The resulting cDNA was diluted to a final concentration of $20 \mathrm{ng} / \mu \mathrm{l}$. The qRT-PCR primers used in this study are shown in Supplementary Table S2. All qRT-PCR reactions were performed with SYBR Green Supermix (Applied Biosystems, Foster City, CA, U.S.A.), following the manufacturer's instructions. For the unannotated gene $N b P D F 1.2 a$, primers were designed based on the AtPDF1.2a mRNA sequence and used to amplify a homologous fragment in $N$. benthamiana cDNA. Sequencing of the amplicon revealed $98 \%$ sequence identity between $N b P D F 2.1 a$ and AtPDF2.1a (Supplementary Fig. S3); qRT-PCR primers were designed based on the amplicons. Actin was used as an internal control and experiments were carried out in triplicate.

Determining the density of RNA bands. A UVP BioSpectrum 600 Imaging System v.6.8 (Upland, CA, U.S.A.) was used to measure the density of BaMV gRNA bands (approximately $6.4 \mathrm{~kb}$ ). Densities from at least three replicates were averaged and used as an indicator of viral RNA accumulation. In the $a b i$ experiment, the gRNA band was too weak to be detected in the WT. Overexposure resulted in the other abi bands becoming oversaturated, preventing comparison of their densities. Therefore, we used sgRNA2 for this purpose.

\section{Protein analyses.}

Leaves (approximately $0.1 \mathrm{~g}$ ) were ground to fine powder in liquid nitrogen and homogenized by adding a similar volume (approximately $400 \mu \mathrm{l})$ of extraction buffer $(0.1 \mathrm{M}$ glycine$\mathrm{NaOH}$ [pH 9.0], 0.1 M NaCl, $0.5 \mathrm{mM}$ EDTA, 2\% sodium dodecyl sulfate [SDS], and 1\% sodium laurosarcosine) (Várallyay et al. 2010). Samples were boiled for $5 \mathrm{~min}$ and subsequently centrifuged at $13,000 \mathrm{rpm}$ for $5 \mathrm{~min}$. Supernatants were then transferred to new tubes. For BaMV CP detection, crude protein extract $(25 \mu \mathrm{g})$ was loaded with $2 \times$ dye $(1 \mathrm{M}$ Tris, $10 \%$ SDS, $100 \%$ glycerol, and $900 \mathrm{ml}$ of $\beta$-mercaptoethanol in $50 \mathrm{ml}$ of $\mathrm{H}_{2} \mathrm{O}$ ) onto $10 \%$ SDS-polyacrylamide gels for Western blot analysis, and membranes were hybridized with BaMV anti-CP sera (Lin et al. 1992).

\section{ELISA.}

ABA was measured as previously described (Hurng et al. 1994). Briefly, samples were collected at 10 and 16 dpi from Arabidopsis and $N$. benthamiana, respectively, then lyophilized and soaked in $1.5 \mathrm{ml}$ of extraction buffer $(80 \% \mathrm{MeOH}$ and $2 \%$ glacial acetic acid [gAA]) for $24 \mathrm{~h}$, then centrifuged at $5,000 \mathrm{rpm}$ for $10 \mathrm{~min}$. The resulting supernatant $(1 \mathrm{ml})$ was speed vacuumed and the pellet was then dissolved in $1: 1 \mathrm{MeOH}$ and $0.2 \mathrm{M} \mathrm{NH}_{4} \mathrm{H}_{2} \mathrm{PO}_{4}$, before being incubated at $4^{\circ} \mathrm{C}$ for 10 min. The samples were applied to individual polyvinyl-polypyrrolidone columns, and the columns were then treated with $6 \mathrm{ml}$ of double-distilled $\mathrm{H}_{2} \mathrm{O}$. A total of $100 \mu \mathrm{l}$ gAA was added to a final volume of $7 \mathrm{ml}$ and passed though C18 columns, which were then washed with washing buffer $(20 \% \mathrm{MeOH}$ and $2 \%$ gAA). Samples were collected with $4 \mathrm{ml}$ of elution buffer (55\% $\mathrm{MeOH}$ and $2 \% \mathrm{gAA}$ ), and $1 \mathrm{ml}$ was speed-vacuumed (this last step was repeated twice, to obtain two mechanical replicates from the same sample). ABA was detected using the Agdia Phytodetek kit (catalog number PDK 09347/0096). Three biological replicates were performed for each line.

\section{VIGS.}

VIGS was performed as described (Dinesh-Kumar et al. 2003). In brief, an $N b A B A l$ fragment of approximately $250 \mathrm{~kb}$ was amplified from $N$. benthamiana cDNA using the primers GCAGAGTCTAGAACATGCCTTGC and CTTCCGAATTCT CGAGCTGC. A 200-kb NbEDS1 fragment was amplified using GGATAAAACTAGGGACGCAG and GTTTTCTTCCCTGA GTCTAC. An approximately 500-bp $N b A B A 2$ fragment was amplified using CAAGAAGGAACTTGCCGCCATGGA and GCGCAAGGCCAGCAATAGCA, and the $N b P D S$ fragment was amplified using GGTGGGAAGGTAGCTGCATGG and GGGCCGACAGGGTTCACAACC. Fragments were cloned separately into pTRV2 and used to transform Agrobacterium tumefaciens $\mathrm{C} 85 \mathrm{C} 1$. The optical density at $600 \mathrm{~nm}$ was ad- 
justed to 1 , and A. tumefaciens carrying pTRV1 or pTRV2 (with the inserted fragment) was co-infiltrated into approximately 16-day-old $N$. benthamiana leaves (three mature leaves per treatment). Plants infiltrated with pTRV1 and pTRV2-PDS began to show a bleaching phenotype at 6 to $7 \mathrm{dpi}$, which indicated successful knockdown of phytoene desaturase. BaMV RNA (approximately $0.2 \mu \mathrm{g}$ ) was inoculated on three leaves above the infiltrated ones; inoculated leaves were collected for RNA analysis at 7 dpi. To determine the efficiency of VIGS, qRT-PCR was performed with primers targeting sites inside the cloned fragments and RNA extracted from upper leaves.

\section{Infection of $N$. benthamiana with CMV and BaMV.}

Three leaves of each $N$. benthamiana plant were inoculated with $1 \mu \mathrm{g}$ of CMV virion or $0.1 \mu \mathrm{g}$ of BaMV viral RNA. Severe symptoms were observed at $16 \mathrm{dpi}$. At this time point, two systemic leaves were collected from each plant for RNA extraction and ELISA. Three plants were examined for each line. Crude extracts from CMV-infected $N$. benthamiana were used to inoculate Arabidopsis thaliana lines. A. thaliana leaves were collected for CMV CP detection at 10 dpi by Western blot using anti CMV-CP sera (Chung 1988). Experiments were performed in triplicate, with at least three plants examined per line.

\section{ACKNOWLEDGMENTS}

This research was supported, in part, by the Academia Sinica Investigator Award, Taipei, Taiwan. We thank J. Parker (Max-Planck Institute) for providing the eds $1-2$ seed and M. de Torres Zabala (University of Exeter ) for the aao3 seed; P. Verslues and W.-H. Cheng (Institute of Plant and Microbial Biology, Academia Sinica) for providing many of the genetic materials and for their support and criticism of this study; and the Sequencing Core Lab at the Institute of Plant and Microbial Biology, Academia Sinica, for technical assistance.

\section{LITERATURE CITED}

Asselbergh, B., De Vleesschauwer, D., and Hofte, M. 2008. Global switches and fine-tuning-ABA modulates plant pathogen defense. Mol. Plant-Microbe Interact. 21:709-719.

Audran, C., Borel, C., Frey, A., Sotta, B., Meyer, C., Simonneau, T., and Marion-Poll, A. 1998. Expression studies of the zeaxanthin epoxidase gene in Nicotiana plumbaginifolia. Plant Physiol. 118:1021-1028.

Barnes, D., and Mayfield, S. P. 2003. Redox control of posttranscriptional processes in the chloroplast. Antioxid. Redox. Sign 5:89-94.

Broekaert, W. F., Delaure, S. L., De Bolle, M. F., and Cammue, B. P. 2006. The role of ethylene in host-pathogen interactions. Annu. Rev. Phytopathol. 44:393-416.

Chen, L. Z., Shi, Q. T., Ding, R. J., and Hu, D. Y. 2013. The prevalence, awareness, and control of hypertension in Xianghe county of Hebei province. Chin. J. Intern. Med. 52:185-187. (In Chinese)

Cheng, W. H., Chiang, M. H., Hwang, S. G., and Lin, P. C. 2009. Antagonism between abscisic acid and ethylene in Arabidopsis acts in parallel with the reciprocal regulation of their metabolism and signaling pathways. Plant Mol. Biol. 71:61-80.

Chung, B. 1988. Symptom determinant of two Taiwan strains of cucumber mosaic virus is on RNA 3. Bot. Bull. Acad. Sin. 29.

Clarke, S. F., Burritt, D. J., and Guy, P. L. 1998. Influence of plant hormones on virus replication and pathogenesis-related proteins in Phaseolus vulgaris L. infected with white clover mosaic potexvirus. Physiol. Mol. Plant Pathol. 53:195-207.

Creelman, R. A., Bell, E., and Mullet, J. E. 1992. Involvement of a lipoxygenase-like enzyme in abscisic acid biosynthesis. Plant Physiol. 99:1258-1260.

Diaz-Pendon, J. A., Li, F., Li, W. X., and Ding, S. W. 2007. Suppression of antiviral silencing by Cucumber mosaic virus $2 \mathrm{~b}$ protein in Arabidopsis is associated with drastically reduced accumulation of three classes of viral small interfering RNAs. Plant Cell 19:2053-2063.

Dinesh-Kumar, S. P., Anandalakshmi, R., Marathe, R., Schiff, M., and Liu, Y. 2003. Virus-induced gene silencing. Methods Mol. Biol. 236:287294

Finkelstein, R. R. 1994. Mutations at 2 new Arabidopsis ABA response loci are similar to the ABI3 mutations. Plant J. 5:765-771.
Finkelstein, R. R., Wang, M. L., Lynch, T. J., Rao, S., and Goodman, H. M. 1998. The Arabidopsis abscisic acid response locus ABI4 encodes an APETALA 2 domain protein. Plant Cell 10:1043-1054.

Fraser, R. S. S., and Whenham, R. J. 1989. Abscisic-Acid metabolism in tomato plants infected with tobacco mosaic virus-Relationships with growth, symptoms and the Tm-1 gene for TMV resistance. Physiol. Mol. Plant Pathol. 34:215-226.

Ghassemian, M., Nambara, E., Cutler, S., Kawaide, H., Kamiya, Y., and McCourt, P. 2000. Regulation of abscisic acid signaling by the ethylene response pathway in Arabidopsis. Plant Cell 12:1117-1126.

Giraudat, J., Hauge, B. M., Valon, C., Smalle, J., Parcy, F., and Goodman, H. M. 1992. Isolation of the Arabidopsis ABI3 gene by positional cloning. Plant Cell 4:1251-1261.

Gonzalez-Guzman, M., Apostolova, N., Belles, J.M., Barrero, J. M., Piqueras, P., Ponce, M. R., Micol, J. L., Serrano, R., and Rodriguez, P.L. 2002. The short-chain alcohol dehydrogenase ABA2 catalyzes the conversion of xanthoxin to abscisic aldehyde. Plant Cell 14:1833-1846.

Goodin, M. M., Zaitlin, D., Naidu, R. A., and Lommel, S. A. 2008. Nicotiana benthamiana: Its history and future as a model for plant-pathogen interactions. Mol. Plant-Microbe Interact. 21:1015-1026.

Han, S. Y., Kitahata, N., Sekimata, K., Saito, T., Kobayashi, M., Nakashima, K., Yamaguchi-Shinozaki, K., Shinozaki, K., Yoshida, S., and Asami, T. 2004. A novel inhibitor of 9-cis-epoxycarotenoid dioxygenase in abscisic acid biosynthesis in higher plants. Plant Physiol. 135:1574-1582.

Hauser, F., Waadt, R., and Schroeder, J. I. 2011. Evolution of abscisic acid synthesis and signaling mechanisms. Curr. Biol. 21:R346-355.

Huang, Z., Yeakley, J. M., Garcia, E. W., Holdridge, J. D., Fan, J. B., and Whitham, S. A. 2005. Salicylic acid-dependent expression of host genes in compatible Arabidopsis-virus interactions. Plant Physiol. 137:11471159

Hurng, W. P., Lur, H. S., Liao, C. K., and Kao, C. H. 1994. Role of abscisic-acid, ethylene and polyamines in flooding-promoted senescence of tobacco leaves. J. Plant Physiol. 143:102-105.

Hwang, S. G., Chen, H. C., Huang, W. Y., Chu, Y. C., Shii, C. T., and Cheng, W. H. 2010. Ectopic expression of rice OsNCED3 in Arabidopsis increases ABA level and alters leaf morphology. Plant Sci. 178:12-22.

Iriti, M., and Faoro, F. 2008. Abscisic acid is involved in chitosan-induced resistance to Tobacco necrosis virus (TNV). Plant Physiol. Biochem. 46:1106-1111.

Jovel, J., Walker, M., and Sanfacon, H. 2011. Salicylic acid-dependent restriction of Tomato ringspot virus spread in tobacco is accompanied by a hypersensitive response, local RNA silencing, and moderate systemic resistance. Mol. Plant-Microbe Interact. 24:706-718.

Koornneef, M., Reuling, G., and Karssen, C. M. 1984. The isolation and characterization of abscisic-acid insensitive mutants of Arabidopsis thaliana. Physiol. Plant. 61:377-383.

Kovac, M., Muller, A., Milovanovic Jarah, D., Milavec, M., Duchting, P., and Ravnikar, M. 2009. Multiple hormone analysis indicates involvement of jasmonate signaling in the early defense of potato to Potato virus $Y^{\mathrm{NTN}}$. Biol. Plant. 53:195-199.

Leung, J., Merlot, S., and Giraudat, J. 1997. The Arabidopsis ABSCISIC ACID-INSENSITIVE2 (ABI2) and ABI1 genes encode homologous protein phosphatases $2 \mathrm{C}$ involved in abscisic acid signal transduction. Plant Cell 9:759-771.

Lewsey, M., Palukaitis, P., and Carr, J. P. 2009. Plant-virus interactions: defence and counter-defence. Annu. Plant Rev. 34:134-176.

Lin, K. Y., Cheng, C. P., Chang, B. C., Wang, W. C., Huang, Y. W., Lee, Y. S., Huang, H. D., Hsu, Y. H., and Lin, N. S. 2010. Global analyses of small interfering RNAs derived from Bamboo mosaic virus and its associated satellite RNAs in different plants. PloS One 5:e11928.

Lin, N. S., Lin, F. Z., Huang, T. Y., and Hsu, Y. H. 1992. Genome properties of bamboo mosaic virus. Phytopathology 82:731-734.

Lin, N. S., Chai, Y. J., Huang, T. Y., Chang, T. Y., and Hsu, Y.H. 1993. Incidence of bamboo mosaic potexvirus in Taiwan. Plant Dis 77:448-450.

Lin, N. S., Lin, B. Y., Lo, N. W., Hu, C. C., Chow, T. Y., and Hsu, Y. H. 1994. Nucleotide sequence of the genomic RNA of bamboo mosaic potexvirus. J. Gen. Virol. 75:2513-2518.

Lin, P. C., Hwang, S. G., Endo, A., Okamoto, M., Koshiba, T., and Cheng, W. H. 2007. Ectopic expression of ABSCISIC ACID 2/GLUCOSE INSENSITIVE 1 in Arabidopsis promotes seed dormancy and stress tolerance. Plant Physiol. 143:745-758.

Mang, H. G., Qian, W., Zhu, Y., Qian, J., Kang, H. G., Klessig, D. F., and Hua, J. 2012. Abscisic acid deficiency antagonizes high-temperature inhibition of disease resistance through enhancing nuclear accumulation of resistance proteins SNC1 and RPS4 in Arabidopsis. Plant Cell 24:1271-1284

Marco, S., and Levy, D. 1979. Involvement of ethylene in the development of Cucumber mosaic virus-induced chlorotic lesions in cucumber cotyledons. Physiol. Plant Pathol. 14:235-244. 
Marin, E., Nussaume, L., Quesada, A., Gonneau, M., Sotta, B., Hugueney, P., Frey, A., and Marion-Poll, A. 1996. Molecular identification of zeaxanthin epoxidase of Nicotiana plumbaginifolia, a gene involved in abscisic acid biosynthesis and corresponding to the ABA locus of Arabidopsis thaliana. EMBO (Eur. Mol. Biol. Organ.) J. 15:2331-2342.

Munemasa, S., Oda, K., Watanabe-Sugimoto, M., Nakamura, Y., Shimoishi, Y., and Murata, Y. 2007. The coronatine-insensitive 1 mutation reveals the hormonal signaling interaction between abscisic acid and methyl jasmonate in Arabidopsis guard cells. Specific impairment of ion channel activation and second messenger production. Plant Physiol. 143:1398-1407.

Nambara, E., and Marion-Poll, A. 2005. Abscisic acid biosynthesis and catabolism. Annu. Rev. Plant Biol. 56:165-185.

Pieterse, C. M., Leon-Reyes, A., Van der Ent, S., and Van Wees, S. C. 2009. Networking by small-molecule hormones in plant immunity. Nat. Chem. Biol. 5:308-316.

Santner, A., Calderon-Villalobos, L. I., and Estelle, M. 2009. Plant hormones are versatile chemical regulators of plant growth. Nat. Chem. Biol. 5:301-307.

Schwartz, S. H., Tan, B. C., McCarty, D. R., Welch, W., and Zeevaart, J. A 2003. Substrate specificity and kinetics for VP14, a carotenoid cleavage dioxygenase in the ABA biosynthetic pathway. Biochim. Biophys. Acta 1619:9-14.

Seo, M., Peeters, A. J., Koiwai, H., Oritani, T., Marion-Poll, A., Zeevaart, J. A., Koornneef, M., Kamiya, Y., and Koshiba, T. 2000. The Arabidopsis aldehyde oxidase 3 (AAO3) gene product catalyzes the final step in abscisic acid biosynthesis in leaves. Proc. Natl. Acad. Sci. U.S.A 97:12908-12913.

Seo, M., Aoki, H., Koiwai, H., Kamiya, Y., Nambara, E., and Koshiba, T. 2004. Comparative studies on the Arabidopsis aldehyde oxidase (AAO) gene family revealed a major role of AAO3 in ABA biosynthesis in seeds. Plant Cell Physiol. 45:1694-1703.

Sheard, L. B., and Zheng, N. 2009. Plant biology signal advance for abscisic acid. Nature 462:575-576.

Soosaar, J. L., Burch-Smith, T. M., and Dinesh-Kumar, S. P. 2005 Mechanisms of plant resistance to viruses. Nat. Rev. Microbiol. 3:789798.

Spoel, S. H., and Dong, X. N. 2012. How do plants achieve immunity? Defence without specialized immune cells. Nat. Rev. Immunol. 12:89100

Ton, J., Flors, V., and Mauch-Mani, B. 2009. The multifaceted role of ABA in disease resistance. Trends Plant Sci. 14:310-317.

Varallyay, E., Valoczi, A., Agyi, A., Burgyan, J., and Havelda, Z. 2010. Plant virus-mediated induction of miR168 is associated with repression of ARGONAUTE1 accumulation. EMBO (Eur. Mol. Biol. Organ.) J. 29:3507-3519.
Wan, X. R., and Li, L. 2006. Regulation of ABA level and water-stress tolerance of Arabidopsis by ectopic expression of a peanut 9-cisepoxycarotenoid dioxygenase gene. Biochem. Biophys. Res. Commun. 347:1030-1038.

Wang, S.-M., Hou, X.-L., Ying, L., Cao, X.-W., Zhang, S., and Wang, F. 2011. Effects of Turnip mosaic virus (TuMV) on endogenous hormones and transcriptional level of related genes in infected non-heading Chinese cabbage. J. Nanjing Agric. Univ. 5:13-19.

Westwood, J. H., McCann, L., Naish, M., Dixon, H., Murphy, A. M., Stancombe, M. A., Bennett, M. H., Powell, G., Webb, A. A. R., and Carr, J. P. 2013. A viral RNA silencing suppressor interferes with abscisic acid-mediated signalling and induces drought tolerance in Arabidopsis thaliana. Mol. Plant Pathol. 14:158-170.

Whenham, R. J., Fraser, R. S. S., Brown, L. P., and Payne, J. A. 1986. Tobacco-mosaic-virus-induced increase in abscisic-acid concentration in tobacco-leaves - Intracellular location in light and dark-green areas, and relationship to symptom development. Planta 168:592-598.

Woloshen, V., Huang, S., and Li, X. 2011. RNA-binding proteins in plant immunity. J. Pathogens 2011:278697.

Xu, P., Chen, F., Mannas, J. P., Feldman, T., Sumner, L. W., and Roossinck, M. J. 2008. Virus infection improves drought tolerance. New Phytol. 180:911-921.

Yang, C. C., Liu, J. S., Lin, C. P., and Lin, N. S. 1997. Nucleotide sequence and phylogenetic analysis of a bamboo mosaic potexvirus isolate from common bamboo (Bambusa vulgaris McClure). Bot. Bull. Acad. Sin. 38:77-84.

Yasuda, M., Ishikawa, A., Jikumaru, Y., Seki, M., Umezawa, T., Asami, T., Maruyama-Nakashita, A., Kudo, T., Shinozaki, K., Yoshida, S., and Nakashita, H. 2008. Antagonistic interaction between systemic acquired resistance and the abscisic acid-mediated abiotic stress response in Arabidopsis. Plant Cell 20:1678-1692.

Zhang, H., Zhu, X., and Liu, H. 1997. Effect of banana bunchy top virus (BBTV) on endogenous hormone of banana plant. Acta Phytopathol. Sin. 27:79-83.

Zhang, M., Yuan, B., and Leng, P. 2009. Cloning of 9-cis-epoxycarotenoid dioxygenase (NCED) gene and the role of ABA on fruit ripening. Plant Signal. Behav. 4:460-463.

Zhang, X., Yuan, Y. R., Pei, Y., Lin, S. S., Tuschl, T., Patel, D. J., and Chua, N. H. 2006. Cucumber mosaic virus-encoded 2b suppressor inhibits Arabidopsis Argonaute1 cleavage activity to counter plant defense. Genes Dev. 20:3255-3268.

\section{AUTHOR-RECOMMENDED INTERNET RESOURCE}

Sol Genomics database: solgenomics.net 